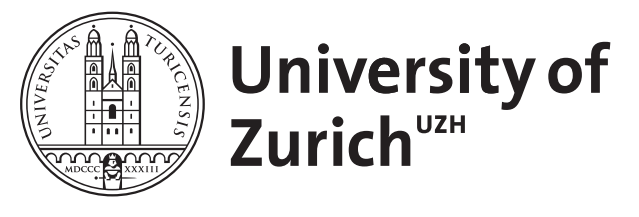

\title{
Massive gravity as a quantum gauge theory
}

\author{
Grigore, D R ; Scharf, G
}

\begin{abstract}
We present a new point of view on the quantization of the massive gravitational field, namely we use exclusively the quantum framework of the second quantization. The Hilbert space of the manygravitons system is a Fock space F+ (Hgraviton) where the one-particle Hilbert space Hgraviton carries the direct sum of two unitary irreducible representations of the Poincaré group corresponding to two particles of mass $\mathrm{m}>0$ and spins 2 and 0 , respectively. This Hilbert space is canonically isomorphic to a space of the type $\operatorname{Ker}(\mathrm{Q}) / \operatorname{Im}(\mathrm{Q})$ where $\mathrm{Q}$ is a gauge charge defined in an extension of the Hilbert space Hgraviton generated by the gravitational field $\mathrm{h}$ and some ghosts fields $\mathrm{u}, \tilde{\mathrm{u}}$ (which are vector Fermi fields) and v (which is a vector Bose field). Then we study the self interaction of massive gravity in the causal framework. We obtain a solution which goes smoothly to the zero-mass solution of linear quantum gravity up to a term depending on the bosonic ghost field. This solution depends on two real constants as it should be; these constants are related to the gravitational constant and the cosmological constant. In the second order of the perturbation theory we do not need a Higgs field, in sharp contrast to Yang-Mills theory
\end{abstract}

DOI: https://doi.org/10.1007/s10714-005-0092-1

Posted at the Zurich Open Repository and Archive, University of Zurich

ZORA URL: https://doi.org/10.5167/uzh-155821

Journal Article

Published Version

Originally published at:

Grigore, D R; Scharf, G (2005). Massive gravity as a quantum gauge theory. General Relativity and Gravitation, 37(6):1075-1096.

DOI: https://doi.org/10.1007/s10714-005-0092-1 


\title{
RESEARCH ARTICLE
}

\author{
D. R. Grigore - G. Scharf
}

\section{Massive gravity as a quantum gauge theory}

Received: 12 October 2004 / Published online: 2 June 2005

(C) Springer-Verlag 2005

Abstract We present a new point of view on the quantization of the massive gravitational field, namely we use exclusively the quantum framework of the second quantization. The Hilbert space of the many-gravitons system is a Fock space $\mathcal{F}^{+}\left(\mathrm{H}_{\text {graviton }}\right)$ where the one-particle Hilbert space $\mathrm{H}_{\text {graviton }}$ carries the direct sum of two unitary irreducible representations of the Poincaré group corresponding to two particles of mass $m>0$ and spins 2 and 0 , respectively. This Hilbert space is canonically isomorphic to a space of the type $\operatorname{Ker}(Q) / \operatorname{Im}(Q)$ where $Q$ is a gauge charge defined in an extension of the Hilbert space $\mathcal{H}_{\text {graviton }}$ generated by the gravitational field $h_{\mu \nu}$ and some ghosts fields $u_{\mu}, \tilde{u}_{\mu}$ (which are vector Fermi fields) and $v_{\mu}$ (which is a vector Bose field).

Then we study the self interaction of massive gravity in the causal framework. We obtain a solution which goes smoothly to the zero-mass solution of linear quantum gravity up to a term depending on the bosonic ghost field. This solution depends on two real constants as it should be; these constants are related to the gravitational constant and the cosmological constant. In the second order of the perturbation theory we do not need a Higgs field, in sharp contrast to Yang-Mills theory.

Keywords Gravitational field · Quantum gravity · Gauge field theories

D. R. Grigore

Department of Theor. Phys., Inst. Atomic Phys., Bucharest-Măgurele, P.O. Box MG 6, ROMÂNIA

E-mail: grigore@theor1.theory.nipne.ro, grigore@theory.nipne.ro

G. Scharf $(\varangle)$

Institute of Theor. Phys., Univ Zürich, Winterthurerstr 190, 8057 Zürich, SWITZERLAND

E-mail: scharf@physik.unizh.ch 


\section{Introduction}

The quantization of gravity is an old standing problem of quantum field theory. The solution of this problem in full generality is a highly non-trivial problem which seems to be extremely complicated. (See however the papers of Ashtekar and collaborators [1, 32]). In [13] and [14-17, 29] this problem was addressed for the linear gravitational field of zero mass. Among the pioneering works in this approach we mention $[8,19,23,24,26,33]$. Using the result of this analysis many computations have been done in the literature (see $[3,4,20,35,36]$ ).

One possible way to perform the quantization of the asymptotic gravitational field is to linearize the classical theory of gravitation using the so called Goldberg variables $[10,18]$ and then to apply straightforward quantization of the resulting free field theory. Because of the gauge invariance of the theory (which in this case is the invariance under general coordinates transformations) one obtains a constrained system and one tries to use a Bleuler-Gupta type formalism, that is to start with a Hilbert space endowed with a sesqui-linear non-degenerate form and select the physical states as a subspace of the type $Q_{A} \Phi=0, A=1, \ldots, N$.

A related idea is to extend the Fock space to an auxiliary Hilbert space $\mathcal{H}^{\text {gh }}$ including some fictitious fields, called ghosts, and construct a gauge charge (i.e. an operator $Q$ verifying $Q^{2}=0$ ) such that the physical Hilbert space is $\mathcal{H}_{\text {phys }} \equiv \operatorname{Ker}(Q) / \operatorname{Im}(Q)$ (see for instance [24] and references quoted there). As a result of this procedure, it is asserted that the graviton, i.e. the elementary quantum particle must be a massless spin 2 particle. The construction of the gauge charge relies heavily on classical field theory arguments, because one tries to obtain for the quantum gauge transformations expressions of the same type as the general coordinates transformations appearing in general relativity. This invariance is then promoted to a quantum gauge invariance which should be implemented by the commutator with the gauge charge $Q$.

It is an interesting problem to consider the case of massive gravity. This case was analyzed many years ago [5, 34]. In [5] it is argued that even the quantization of the massive spin 2 field is problematic in the sense that no smooth limit $m \mapsto 0$ exists. Some recent interest on this problem exists [2, 21, 25, 27] and [6].

We will show here that one can perform the quantization in such a way that this limit is smooth. One finds out that the massive graviton has a scalar partner of the same mass $m$. The construction is done in the spirit of [13].

We also mention that a rigorous construction of the Hilbert space of the manygravitons system is indispensable for the construction of the corresponding $S$ matrix in perturbation theory in the sense of Bogoliubov. This construction emphasizes the basic rôle of causality in quantum field theory. We obtain a solution for the interaction Lagrangian (the first-order chronological product) which goes smoothly for $m \searrow 0$ into the solution appearing in [29].

The solution we obtain, up to second order of the perturbation theory, coincides with the result of the perturbative development of the Einstein-Hilbert Lagrangian with cosmological constant, if we make the identification $\Lambda=2 m^{2}$ and use Goldberg variables (see the Conclusions). We remark that in the second order of the perturbation theory we do not need a Higgs field as in the case of Yang-Mills fields. For this reason it seems to be impossible to find our massive spin 2 gauge theory by means of the conventional Higgs mechanism. 


\section{The quantization of the asymptotic massive gravitational field}

One defines the graviton as a certain unitary irreducible representation of the Poincaré group corresponding to zero mass and helicity 2. In the case of massive gravity one should use the representation of positive mass $m$ and spin 2 . These representation can be explicitly described using the formalism of Hilbert space bundles, as presented for instance in [34], Chap. VI.7 thm 6.20. Let us denote by $H_{\mathrm{gr}}^{(m)}$ the one-particle Hilbert space of the graviton of mass $m$. The ensemble of many gravitons is usually described by the associated Fock space $\mathcal{F}_{\text {graviton }}=\mathcal{F}^{(+)}\left(H_{\mathrm{gr}}^{(m)}\right)$ where the upper + sign indicates that the gravitons are assumed to be Bosons according to the well-known spin-statistics theorem. The Hilbert space $\mathcal{F}_{\text {graviton }}$ is not very suitable for the construction of the perturbative series of the scattering matrix $S$ in the sense of Bogoliubov. The way out is to construct a larger Hilbert space $\mathcal{H}$ where unphysical degrees of freedom are present. In this Hilbert space a (gravitational) gauge charge $Q$ acts which should be chosen such that it squares to zero $Q^{2}=0$; in this case it makes sense to consider the factor space $\mathcal{H}_{\text {phys }} \equiv \operatorname{Ker}(Q) / \operatorname{Im}(Q)$ which should be canonically isomorphic to $\mathcal{F}_{\text {graviton }}$.

Let us describe this construction. We use in this paper the following notations. The upper hyperboloid of mass $m \geq 0$ is by definition $X_{m}^{+} \equiv\left\{p \in \mathbb{R}^{4} \mid\|p\|^{2}=\right.$ $\left.m^{2}\right\}$; it is a Borel set with the Lorentz invariant measure $d \alpha_{m}^{+}(p) \equiv \frac{d \mathbf{p}}{2 \omega(\mathbf{p})}$. Here the conventions are the following: $\|\cdot\|$ is the Minkowski norm defined by $\|p\|^{2} \equiv p \cdot p$ and $p \cdot q$ is the Minkowski bilinear form: $p \cdot q \equiv p_{0} q_{0}-\mathbf{p} \cdot \mathbf{q}$; by $\eta_{\mu \nu}$ we denote the corresponding flat Minkowski matrix with diagonal elements $1,-1,-1,-1$. If $\mathbf{p} \in \mathbb{R}^{3}$ we define $\tau(\mathbf{p}) \in X_{m}^{+}$according to $\tau(\mathbf{p}) \equiv(\omega(\mathbf{p}), \mathbf{p}), \quad \omega(\mathbf{p}) \equiv$ $\sqrt{\mathbf{p}^{2}+m^{2}}$.

First we consider the zero mass case $m=0[13,29]$.

- One generates the Hilbert space $\mathcal{H}$ by applying the fields $H_{\mu \nu}, u_{\rho}, \tilde{u}_{\rho}, \Phi$ on the vacuum $\Omega \in \mathcal{H}$ (the rigorous construction is based on the Borchers algebra); these fields are of null mass:

$$
\partial^{2} H_{\mu \nu}(x)=0 \quad \partial^{2} u_{\rho}(x)=0 \quad \partial^{2} \tilde{u}_{\rho}(x)=0 \quad \partial^{2} \Phi(x)=0 .
$$

- $H_{\mu \nu}$ is symmetric and traceless:

$$
H_{\mu \nu}=H_{\nu \mu} \quad H_{\mu}^{\mu}=0 .
$$

- The field $\Phi$ is scalar and $H_{\mu \nu}, u_{\rho}, \tilde{u}_{\rho}$ have obvious tensor and vector properties . This implies the existence of a projective unitary representation $\mathcal{U}_{g}$ of the Poincaré group $\mathcal{P}$ acting in the Hilbert space $\mathcal{H}$.

- The fields $H_{\mu \nu}, \Phi$ are Bosons and $u_{\rho}, \tilde{u}_{\rho}$ are Fermions.

- The causal commutation relations of these fields are

$$
\begin{aligned}
{\left[H_{\rho \sigma}(x), H_{\lambda \omega}(y)\right] } & =-\frac{i}{2}\left(\eta_{\rho \lambda} \eta_{\sigma \omega}+\eta_{\rho \omega} \eta_{\sigma \lambda}-\frac{1}{2} \eta_{\rho \sigma} \eta_{\lambda \omega}\right) D_{0}(x-y) \times \mathbf{1} \\
\left\{u_{\mu}(x), \tilde{u}_{\nu}(y)\right\} & =i \eta_{\mu \nu} D_{0}(x-y) \mathbf{1} \\
{[\Phi(x), \Phi(y)] } & =i D_{0}(x-y) \mathbf{1}
\end{aligned}
$$


and the other (anti)commutators are zero; in particular all Bose fields commute with all Fermi fields. Here

$$
D_{m}(x)=D_{m}^{(+}(x)+D_{m}^{(-)}(x)
$$

is the Pauli-Jordan distribution of mass $m \geq 0$ and $D_{m}^{( \pm)}(x)$ are given by:

$$
D_{m}^{( \pm)}(x) \equiv \pm \frac{i}{(2 \pi)^{3 / 2}} \int_{X_{m}^{+}} d \alpha_{m}^{+}(p) e^{\mp i p \cdot x} .
$$

- In this Hilbert space there exists a sesqui-linear form (not positively defined) $<\cdot, \cdot>$ such that we have

$$
H_{\mu \nu}^{\dagger}=H_{\mu \nu} \quad u_{\mu}^{\dagger}=u_{\mu} \quad \tilde{u}_{\mu}^{\dagger}=-\tilde{u}_{\mu} \quad \Phi^{\dagger}=\Phi
$$

where by $\dagger$ we mean the adjoint with respect to $<\cdot, \cdot>$

- The operator $Q$ is well defined through the relations

$$
\begin{gathered}
Q \Omega=0 \\
{\left[Q, H_{\mu \nu}\right]=-\frac{i}{2}\left(\partial_{\mu} u_{\nu}+\partial_{\nu} u_{\mu}-\frac{1}{2} \eta_{\mu \nu} \partial_{\rho} u_{\sigma}\right) \quad[Q, \Phi]=\frac{i}{2} \partial^{\rho} u_{\rho}} \\
\left\{Q, u_{\mu}\right\}=0, \quad\left\{Q, \tilde{u}_{\mu}\right\}=i\left(\partial^{\nu} H_{\mu \nu}+\frac{1}{2} \partial_{\mu} \Phi\right) .
\end{gathered}
$$

In these conditions one can prove that:

- The operator $Q$ is well defined; for this one has to check the validity of the Jacoby identity:

$$
[b(x),\{f(y), Q\}]+\{f(y),[Q, b(x)]\}=0
$$

where $b$ and $f$ are generic Bose (resp. Fermi) fields.

- The following relations are verified:

$$
\begin{aligned}
Q^{2} & =0 \\
\mathcal{U}_{g} Q & =Q \mathcal{U}_{g}, \quad \forall g \in \mathcal{P} .
\end{aligned}
$$

From (2.11) we have

$$
\operatorname{Im}(Q) \subset \operatorname{Ker}(Q)
$$

so it makes sense to consider the factor space $\operatorname{Ker}(Q) / \operatorname{Im}(Q)$. One can prove that the sesqui-linear form $<\cdot, \cdot>$ induces a strictly positively defined scalar product on $\overline{\operatorname{Ker}(Q) / \operatorname{Im}(Q)}$ and we have a canonical isomorphism $\overline{\operatorname{Ker}(Q) / \operatorname{Im}(Q)} \sim \mathcal{F}_{\text {graviton }}$.

The preceding construction presented in detail in [13] justifies the consideration of the auxiliary Hilbert space $\mathcal{H}$ as a playground for the perturbation theory. The fields $u_{\rho}, \tilde{u}_{\rho}, \Phi$ are called ghost fields and the operator $Q$ is the gauge charge. A simplification of the preceding formalism is the consideration of the new field

$$
h_{\mu \nu} \equiv H_{\mu \nu}+\frac{1}{2} \eta_{\mu \nu} \Phi
$$


which is self-adjoint in the sense

$$
h_{\mu \nu}(x)^{\dagger}=h_{\mu \nu}(x)
$$

but is not traceless anymore and the causal commutation relations are:

$$
\begin{aligned}
{\left[h_{\rho \sigma}(x), h_{\lambda \omega}(y)\right]=} & -\frac{i}{2}\left(\eta_{\rho \lambda} \eta_{\sigma \omega}+\eta_{\rho \omega} \eta_{\sigma \lambda}-\eta_{\rho \sigma} \eta_{\lambda \omega}\right) \\
& \times D_{0}(x-y) \times \mathbf{1} .
\end{aligned}
$$

We can easily prove that the preceding definition of the gauge charge is equivalently described by (2.7) and:

$$
\begin{aligned}
{\left[Q, h_{\mu \nu}\right] } & =-\frac{i}{2}\left(\partial_{\mu} u_{\nu}+\partial_{\nu} u_{\mu}-\eta_{\mu \nu} \partial_{\rho} u^{\rho}\right) \\
\left\{Q, u_{\mu}\right\} & =0, \quad\left\{Q, \tilde{u}_{\mu}\right\}=i \partial^{\nu} h_{\mu \nu}
\end{aligned}
$$

so one can consider that the Hilbert space $\mathcal{H}$ is generated by the fields $h_{\mu \nu}, u_{\rho}, \tilde{u}_{\rho}$ with the properties described above.

We now turn to the massive gravitational field. One notices from the very beginning that in the case $m>0$ the gauge charge defined by (2.17) does not square to zero anymore. One can try to correct this feature as in the case of the massive vector field (see for instance [29]) by introducing a new ghost field $v_{\mu}$ which is a vector field. The one modifies the preceding scheme as follows:

- One generates the Hilbert space $\mathcal{H}$ by applying the fields $h_{\mu \nu}, u_{\rho}, \tilde{u}_{\rho}, v_{\mu}$; on the vacuum $\Omega \in \mathcal{H}$; all these fields are of mass $m$ :

$$
\begin{array}{rr}
\left(\partial^{2}+m^{2}\right) h_{\mu \nu}(x)=0 & \left(\partial^{2}+m^{2}\right) u_{\rho}(x)=0 \\
\left(\partial^{2}+m^{2}\right) \tilde{u}_{\rho}(x)=0 & \left(\partial^{2}+m^{2}\right) v_{\mu}(x)=0
\end{array}
$$

- $h_{\mu \nu}$ is symmetric:

$$
h_{\mu \nu}=h_{\nu \mu}
$$

- The fields $h_{\mu \nu}, u_{\rho}, \tilde{u}_{\rho}, v_{\mu}$ have obvious tensor and vector properties

- The fields $h_{\mu \nu}, v_{\mu}$ are Bosons and $u_{\rho}, \tilde{u}_{\rho}$ are Fermions

- The causal commutation relations of these fields are

$$
\begin{aligned}
{\left[h_{\rho \sigma}(x), h_{\lambda \omega}(y)\right] } & =-\frac{i}{2}\left(\eta_{\rho \lambda} \eta_{\sigma \omega}+\eta_{\rho \omega} \eta_{\sigma \lambda}-\eta_{\rho \sigma} \eta_{\lambda \omega}\right) D_{m}(x-y) \times \mathbf{1} \\
\left\{u_{\mu}(x), \tilde{u}_{\nu}(y)\right\} & =i \eta_{\mu \nu} D_{a m}(x-y) \mathbf{1} \\
{\left[v_{\mu}(x), v_{\rho}(y)\right] } & =\frac{i}{2} D_{m}(x-y) \mathbf{1}
\end{aligned}
$$

and the other (anti)commutators are zero

- In this Hilbert space there exists a sesqui-linear form (not positively defined) $<\cdot, \cdot>$ such that we have

$$
h_{\mu \nu}^{\dagger}=h_{\mu \nu} \quad u_{\mu}^{\dagger}=u_{\mu} \quad \tilde{u}_{\mu}^{\dagger}=-\tilde{u}_{\mu} \quad v_{\mu}^{\dagger}=v_{\mu}
$$

where by $\dagger$ we mean the adjoint with respect to $\langle\cdot, \cdot>$ 
- The operator $Q$ is well defined through the relations(2.7 ) and

$$
\begin{aligned}
{\left[Q, h_{\mu \nu}\right] } & =-\frac{i}{2}\left(\partial_{\mu} u_{\nu}+\partial_{\nu} u_{\mu}-\eta_{\mu \nu} \partial_{\rho} u_{\sigma}\right) \quad\left[Q, v_{\mu}\right]=-\frac{i m}{2} u_{\mu} \\
\left\{Q, u_{\mu}\right\} & =0, \quad\left\{Q, \tilde{u}_{\mu}\right\}=i\left(\partial^{\nu} h_{\mu \nu}+m v_{\mu}\right) .
\end{aligned}
$$

In these conditions one can prove that the operator $Q$ is well defined because of the validity of the Jacobi identity (2.9) and we also have (2.11) and (2.11), so again it makes sense to consider the factor space $\overline{\operatorname{Ker}(Q) / \operatorname{Im}(Q)}$. One can prove in this case also that the sesqui-linear form $<\cdot, \cdot\rangle$ induces a strictly positively defined scalar product on this factor space. However, in this case a modification of the zero mass scheme appears. The one-particle Hilbert corresponding to $\overline{\operatorname{Ker}(Q) / \operatorname{Im}(Q)}$ is $\mathrm{H}^{[m, 2]} \oplus \mathrm{H}^{[m, 0]}$ i.e. it describes two particles of mass $m$ and spins 2 and one of spin 0 , respectively. In other words, we have $\overline{\operatorname{Ker}(Q) / \operatorname{Im}(Q)}=\mathcal{F}_{\text {gravition }} \oplus$ $\mathcal{F}_{\text {scalar. It seems impossible to construct a gauge structure such that the scalar }}$ partner of the graviton is eliminated, so in this paper we will accept that such a particle does exists. It remains to be investigated whether the scalar partner of the graviton with a tiny mass leads to phenomenological problems.

Sometimes it is convenient to generalize the expression of the new field (2.13) in the sense:

$$
h_{\mu \nu}^{(\alpha)} \equiv H_{\mu \nu}+\frac{1}{2} \alpha \eta_{\mu \nu} \Phi
$$

with $\alpha \in \mathbb{R}^{*}$. The causal commutation relations are for this field:

$$
\begin{aligned}
{\left[h_{\rho \sigma}^{(\alpha)}(x), h_{\lambda \omega}^{(\alpha)}(y)\right]=- } & \frac{i}{2}\left(\eta_{\rho \lambda} \eta_{\sigma \omega}+\eta_{\rho \omega} \eta_{\sigma \lambda}-\frac{1+\alpha^{2}}{2} \eta_{\rho \sigma} \eta_{\lambda \omega}\right) \\
& D_{m}(x-y) \times \mathbf{1} .
\end{aligned}
$$

We can prove that the definition of the gauge charge is equivalently described by (2.7) and:

$$
\begin{gathered}
{\left[Q, h_{\mu \nu}^{(\alpha)}\right]=-\frac{i}{2}\left(\partial_{\mu} u_{\nu}+\partial_{\nu} u_{\mu}-\frac{1+\alpha}{2} \eta_{\mu \nu} \partial_{\rho} u^{\rho}\right),} \\
\left\{Q, u_{\mu}\right\}=0, \quad\left\{Q, \tilde{u}_{\mu}\right\}=i\left(\partial^{\nu} h_{\mu \nu}^{(\alpha)}+\frac{1-\alpha}{4 \alpha} \partial_{\mu} h^{(\alpha)}+m v_{\mu}\right)
\end{gathered}
$$

and

$$
\left[Q, v_{\mu}\right]=-\frac{i m}{2} u_{\mu}
$$

here

$$
h^{(\alpha)} \equiv \eta^{\mu \nu} h_{\mu \nu}^{(\alpha)}
$$

The choice (2.13) correspond to $\alpha=1$. Let us consider the choice $\alpha=-1$. Then the preceding relations for

$$
\hat{h}_{\mu \nu}=h_{\mu \nu}^{(-1)}
$$


become:

$$
\begin{aligned}
& {\left[\hat{h}_{\rho \sigma}(x), \hat{h}_{\lambda \omega}(y)\right]=}-\frac{i}{2}\left(\eta_{\rho \lambda} \eta_{\sigma \omega}+\eta_{\rho \omega} \eta_{\sigma \lambda}-\eta_{\rho \sigma} \eta_{\lambda \omega}\right) \\
& \times D_{m}(x-y) \times \mathbf{1} . \\
& {\left[Q, \hat{h}_{\mu \nu}\right] }=-\frac{i}{2}\left(\partial_{\mu} u_{\nu}+\partial_{\nu} u_{\mu}\right), \\
&\left\{Q, u_{\mu}\right\}=0, \quad\left\{Q, \tilde{u}_{\mu}\right\}=i\left(\partial^{\nu} \hat{h}_{\mu \nu}-\frac{1}{2} \partial_{\mu} \hat{h}+m v_{\mu}\right)
\end{aligned}
$$

and

$$
\left[Q, v_{\mu}\right]=-\frac{i m}{2} u_{\mu} .
$$

This choice seems to appear naturally in the classical framework of gravity with an non-zero cosmological constant, if one expands the metric $g_{\mu \nu}$ around Minkowski background in the form

$$
g_{\mu \nu}=\eta_{\mu \nu}+\kappa \hat{h}_{\mu \nu}
$$

(see the Conclusions). However, from the quantum point of view the value of $\alpha$ is irrelevant: all choices are good for the description of the physical Hilbert space.

We remark also that the massless limit problem mentioned in [5] has a very simple explanation according to the preceding observation: in [5] one uses different values of the parameter $\alpha$ for the case $m=0$ and $m>0$ respectively. The correct procedure is to use the same value of $\alpha$ in both cases.

The construction of observables can be done in the usual way. We denote by $\mathcal{W}$ the linear space of all Wick monomials on the Fock space $\mathcal{H}^{g h}$ i.e. containing the fields $h_{\mu \nu}(x), u_{\mu}(x), \tilde{u}_{\mu}(x)$ and $v_{\mu}(x)$. If $M$ is such a Wick monomial, we define by $g h_{ \pm}(M)$ the degree in $\tilde{u_{\mu}}$ (resp. in $u_{\mu}$ ). The total degree of $M$ is

$$
\operatorname{deg}(M) \equiv g h_{+}(M)+g h_{-}(M) .
$$

The ghost number is, by definition, the expression:

$$
g h(M) \equiv g h_{+}(M)-g h_{-}(M) .
$$

If $M \in \mathcal{W}$ let us define the operator:

$$
d_{Q} M \equiv Q M-(-1)^{g h(M)} M Q
$$

on monomials $M$ and extend it by linearity to the whole $\mathcal{W}$. Then $d_{Q} M \in \mathcal{W}$ and

$$
g h\left(d_{Q} M\right)=g h(M)-1 .
$$

The operator $d_{Q}: \mathcal{W} \rightarrow \mathcal{W}$ is called the gauge variation; the properties of this object are summarized in the following relations:

$$
d_{Q}^{2}=0
$$




$$
\begin{gathered}
d_{Q} h_{\mu \nu}=-\frac{i}{2}\left(\partial_{\mu} u_{\nu}+\partial_{\nu} u_{\mu}-\eta_{\mu \nu} \partial_{\rho} u^{\rho}\right) \\
d_{Q} u_{\mu}=0 \quad d_{Q} \tilde{u}_{\mu}=i\left(\partial^{v} h_{\mu \nu}+m v_{\mu}\right) \\
d_{Q} v_{\mu}=-\frac{i m}{2} u_{\mu}(x) . \\
d_{Q}(M N)=\left(d_{Q} M\right) N+(-1)^{g h(M)} M\left(d_{Q} N\right), \quad \forall M, N \in \mathcal{W} .
\end{gathered}
$$

If $\Psi \in \mathcal{H}$ then we denote by $[\Psi]$ the corresponding equivalence class from the factor space $\overline{\operatorname{Ker}(Q) / \operatorname{Im}(Q)}$. If $O: \mathcal{H}^{g h} \rightarrow \mathcal{H}^{g h}$ verifies the condition

$$
d_{Q} O=0
$$

then it induces a well defined operator $[O]$ on the factor space $\overline{\operatorname{Ker}(Q) / \operatorname{Im}(Q)}$.

Moreover, in this case the following formula is true for the matrix elements of the factorized operator $[O]$ :

$$
([\Psi],[O][\Phi])=(\Psi, O \Phi) .
$$

This kind of observables on the physical space will also be called gauge invariant observables. An operator $O: \mathcal{H}^{g h} \rightarrow \mathcal{H}^{g h}$ induces a gauge invariant observables if and only if it verifies:

$$
\left.d_{Q} O\right|_{\operatorname{Ker}(Q)}=0
$$

Not all operators verifying the condition (2.40) are interesting. In fact, the operators of the type $d_{Q} O$ are inducing a null operator on the factor space; explicitly we have:

$$
\left[d_{Q} O\right]=0 .
$$

In the framework of perturbative quantum field theory [29] the basic objects are the chronological products $T_{n}\left(x_{1}, \ldots, x_{n}\right)$; these are some distribution-valued operators acting in the Hilbert space $\mathcal{H}$ and subject to a set of axioms due to Bogoliubov. The chronological products should leave invariant the physical Hilbert space $\overline{\operatorname{Ker}(Q) / \operatorname{Im}(Q)}$ at least in the adiabatic limit. This leads to a new axiom of factorization in the adiabatic limit:

$$
\left.\lim _{\epsilon \searrow 0} d_{Q} \int_{\mathbb{R}^{4}} d x T_{n}\left(x_{1}, \ldots, x_{n}\right) g(\epsilon x)\right|_{\operatorname{Ker}(Q)}=0, \quad \forall n \in \mathbb{N}^{*}
$$

where $g(x)$ is test function. If the limit exists, then the preceding relation is equivalent to

$$
d_{Q} T_{n}\left(x_{1}, \ldots, x_{n}\right)=i \sum_{l=1}^{n} \frac{\partial}{\partial x_{l}^{\mu}} T_{n / l}^{\mu}\left(x_{1}, \ldots, x_{n}\right), \quad \forall n \in \mathbb{N}^{*} .
$$

If infrared divergences cannot be avoided, the one can consider (2.44) at the heuristic level and impose (2.45) as the new postulate as it is done in [28]. In particular we have for $n=1$

$$
d_{Q} T_{1}(x)=i \frac{\partial}{\partial x^{\mu}} T^{\mu}(x)
$$


for some Wick polynomials $T^{\mu}(x)$. The derivation of the most general expression of $T_{1}$ can be done in the original variables $H_{\mu \nu}, \Phi, u_{\mu}, \tilde{u}_{\mu}, v_{\mu}$ or with $h_{\mu \nu}^{(\alpha)}$ for any values of $\alpha$. If we change the fields, we must correspondingly change the expression of the operator $d_{Q}$ and the final result should be the same. In formulæ:

$$
\begin{aligned}
d_{Q} T_{1}\left(H_{\mu \nu}, \ldots\right) & =i \frac{\partial}{\partial x^{\mu}} T^{\mu}\left(H_{\mu \nu}, \ldots\right) \Leftrightarrow d_{Q} T_{1}\left(h_{\mu \nu}^{(\alpha)}, \ldots\right) \\
& =i \frac{\partial}{\partial x^{\mu}} T^{\mu}\left(h_{\mu \nu}^{(\alpha)}, \ldots\right)
\end{aligned}
$$

for any $\alpha$.

\section{Gauge invariance in the first order of perturbation theory}

In view of the discussion from the preceding section it is natural to discard from the interaction Lagrangian (the first-order chronological product $T_{1}$ ) expressions of the type

$$
d_{Q} B+\partial_{\mu} B^{\mu}, \quad g h(B)=-1, g h\left(B^{\mu}\right)=0 ;
$$

we call such an expression a trivial coupling. If the difference of two couplings is a trivial one then we call them equivalent. In this section we prove the following

Theorem 3.1 Let us consider the most general Wick polynomial T tri-linear in the fields $H_{\mu \nu}, \Phi, u_{\mu}, \tilde{u}_{\mu}, v_{\mu}$ verifying the following conditions:

$$
\begin{aligned}
\mathcal{U}_{g} T & =T \mathcal{U}_{g}, \quad \forall g \in \mathcal{P} \\
g h(T) & =0 \\
3 & \leq \operatorname{deg}(T) \leq 5
\end{aligned}
$$

and the gauge invariance condition (2.46). Then $T$ is equivalent to the following expression:

$$
T=a T^{(a)}+b T^{(b)} \quad a, b \in \mathbb{R}
$$

where

$$
\begin{aligned}
T^{(a)} \equiv & {\left[-2 H^{\mu v}\left(\partial_{\mu} H_{\rho \sigma}\right)\left(\partial_{\nu} H^{\rho \sigma}\right)-4 H^{\mu \nu}\left(\partial^{\alpha} H^{\rho \mu}\right)\left(\partial^{\rho} H_{\alpha v}\right)\right.} \\
& +2 \Phi\left(\partial^{\mu} H^{\rho \sigma}\right)\left(\partial_{\rho} H_{\mu \sigma}\right) \\
& +4 \Phi H^{\rho \sigma}\left(\partial_{\rho} \partial^{v} H_{v \sigma}\right)+\Phi^{2}\left(\partial_{\mu} \partial_{\nu} H^{\mu \nu}\right) \\
& +\left(\partial^{\mu} \Phi\right)\left(\partial^{v} \Phi\right) H_{\mu \nu}+4 H^{\mu v} u^{\rho}\left(\partial_{\mu} \partial_{\nu} \tilde{u}_{\rho}\right)+4\left(\partial_{\mu} H^{\mu v}\right) u^{\rho}\left(\partial_{\nu} \tilde{u}_{\rho}\right) \\
& \left.+4\left(\partial_{\mu} H^{\mu \nu}\right) u^{\rho}\left(\partial_{\rho} \tilde{u}_{v}\right)+2\left(\partial^{\mu} \Phi\right) u^{\rho}\left(\partial_{\rho} \tilde{u}_{\mu}\right)+4 H^{\mu \nu}\left(\partial_{\mu} v_{\rho}\right)\left(\partial_{\nu} v^{\rho}\right)\right] \\
& -4 m\left(\partial_{\mu} v_{v}\right) u^{\mu} \tilde{u}^{v}+m^{2}\left(\frac{2}{3} H^{\mu v} H_{\mu \rho} H_{\nu}{ }^{\rho}+\frac{1}{2} H^{\mu \nu} H_{\mu \nu} \Phi-\frac{3}{4} \Phi^{3}\right. \\
& \left.-\Phi u^{\mu} \tilde{u}_{\mu}+\Phi v^{\mu} v_{\mu}\right)
\end{aligned}
$$


and

$$
\begin{aligned}
T^{(b)} \equiv & -H^{\mu v}\left(\partial_{\mu} v_{\rho}\right)\left(\partial_{\nu} v^{\rho}\right)+2 H^{\mu v}\left(\partial_{\mu} v_{\nu}\right)\left(\partial_{\rho} v^{\rho}\right)-2 H^{\mu v}\left(\partial_{\mu} v_{\rho}\right)\left(\partial^{\rho} v^{v}\right) \\
& +m\left[H^{\mu v}\left(\partial_{\rho} H_{\mu \nu}\right) v^{\rho}-2 H^{\mu v}\left(\partial_{\nu} H_{\mu \rho}\right) v^{\rho}+H^{\mu v}\left(\partial_{\mu} \Phi\right) v_{v}\right. \\
& +\left(\partial_{\rho} v^{\rho}\right) u_{\mu} \tilde{u}^{\mu}-\frac{1}{2}\left(\partial_{\mu} v_{\nu}\right) u^{\mu} \tilde{u}^{v}+\left(\partial_{\nu} v_{\mu}\right) u^{\mu} \tilde{u}^{v}+v^{v} u^{\mu}\left(\partial_{\nu} \tilde{u}_{\mu}\right) \\
& \left.+v_{\mu} u^{\mu}\left(\partial^{v} \tilde{u}_{\mu}\right)-\frac{1}{2} v^{v} u^{\mu}\left(\partial_{\mu} \tilde{u}_{v}\right)+2 v_{\mu} v_{\nu}\left(\partial^{\mu} v^{\nu}\right)\right] \\
& +m^{2}\left(-\frac{1}{3} H^{\mu v} H_{\mu \rho} H_{\nu}{ }^{\rho}-H^{\mu v} u_{\mu} \tilde{u}_{v}+\frac{3}{2} H^{\mu v} v_{\mu} v_{v}\right) .
\end{aligned}
$$

The preceding expression has a smooth limit $m \rightarrow 0$.

Proof We make a list of all Wick monomials verifying the conditions (3.3). The condition of Lorentz invariance depends essentially on the dimension of the spacetime. In other dimensions than 4 the list below changes drastically. Also the fact that $H_{\mu \nu}$ is traceless is useful in eliminating many terms. First we have terms which do also appear for the massless case i.e. without the ghost field $v_{\mu}$ namely:

$$
\begin{aligned}
& T^{(1)}=c^{(1)} H^{\mu v} H_{\mu \rho} H_{\nu}{ }^{\rho} \\
& T^{(2)}=c^{(2)} H^{\mu \nu} H_{\mu \nu} \Phi \\
& T^{(3)}=c^{(3)} \Phi^{3} \\
& T^{(4)}=c^{(4)} H^{\mu v} u_{\mu} \tilde{u}_{\nu} \\
& T^{(5)}=c^{(5)} \Phi u^{\mu} \tilde{u}_{\mu} \\
& T^{(6)}=c_{1}^{(6)} H^{\mu \nu}\left(\partial_{\mu} H_{\rho \sigma}\right)\left(\partial_{\nu} H^{\rho \sigma}\right)+c_{2}^{(6)} H^{\mu \nu}\left(\partial_{\rho} H_{\rho \sigma}\right)\left(\partial_{\sigma} H^{\mu \nu}\right) \\
& +c_{3}^{(6)} H^{\mu \nu}\left(\partial_{\mu} H^{\rho \sigma}\right)\left(\partial_{\rho} H_{\nu \sigma}\right) \\
& +c_{4}^{(6)} H^{\mu \nu}\left(\partial_{\mu} H_{\rho \nu}\right)\left(\partial_{\sigma} H^{\sigma \rho}\right)+c_{5}^{(6)} H^{\mu \nu}\left(\partial^{\rho} H_{\rho \mu}\right)\left(\partial^{\sigma} H_{\sigma \nu}\right) \\
& +c_{6}^{(6)} H^{\mu \nu}\left(\partial^{\alpha} H^{\rho \mu}\right)\left(\partial^{\rho} H_{\alpha \nu}\right) \\
& +c_{7}^{(6)} \epsilon_{\mu \rho \alpha \lambda} H^{\mu \nu}\left(\partial^{\lambda} H^{\rho}{ }_{\nu}\right)\left(\partial_{\beta} H^{\alpha \beta}\right)+c_{8}^{(6)} \epsilon_{\mu \rho \alpha \lambda} H^{\mu \nu}\left(\partial^{\lambda} H^{\rho \sigma}\right)\left(\partial_{\sigma} H^{\alpha}{ }_{\nu}\right) \\
& +c_{9}^{(6)} \epsilon_{\mu \rho \alpha \lambda} H^{\mu \nu}\left(\partial^{\lambda} H^{\rho \sigma}\right)\left(\partial_{\nu} H^{\alpha}{ }_{\sigma}\right) \\
& T^{(7)}=c_{1}^{(7)} \Phi\left(\partial_{\mu} H^{\mu \sigma}\right)\left(\partial^{\nu} H_{\nu \sigma}\right)+c_{2}^{(7)} \Phi\left(\partial^{\mu} H^{\rho \sigma}\right)\left(\partial_{\rho} H_{\mu \sigma}\right) \\
& +c_{3}^{(7)} \epsilon_{\mu \nu \rho \alpha} \Phi\left(\partial^{\mu} H^{\rho \sigma}\right)\left(\partial^{v} H^{\alpha}{ }_{\sigma}\right) \\
& T^{(8)}=c^{(8)} \Phi H^{\rho \sigma}\left(\partial_{\rho} \partial^{\nu} H_{\nu \sigma}\right) \\
& T^{(9)}=c^{(9)} \Phi^{2}\left(\partial_{\mu} \partial_{\nu} H^{\mu \nu}\right) \\
& T^{(10)}=c^{(10)}\left(\partial^{\mu} \Phi\right)\left(\partial^{\nu} \Phi\right) H_{\mu \nu}
\end{aligned}
$$


Massive gravity as a quantum gauge theory

1085

$$
\begin{aligned}
& T^{(11)}=c_{1}^{(11)}\left(\partial_{\mu} H^{\mu \nu}\right) u_{\nu}\left(\partial_{\rho} \tilde{u}^{\rho}\right) \\
& +c_{2}^{(11)}\left(\partial_{\mu} H^{\mu v}\right) u^{\rho}\left(\partial_{\nu} \tilde{u}_{\rho}\right)+c_{3}^{(11)}\left(\partial_{\mu} H^{\mu v}\right) u^{\rho}\left(\partial_{\rho} \tilde{u}_{v}\right) \\
& +c_{4}^{(11)}\left(\partial^{\rho} H^{\mu v}\right) u_{\mu}\left(\partial_{\nu} \tilde{u}_{\rho}\right)+c_{5}^{(11)}\left(\partial^{\rho} H^{\mu v}\right) u_{\rho}\left(\partial_{\mu} \tilde{u}_{v}\right) \\
& +c_{6}^{(11)} \epsilon_{\mu \rho \alpha \sigma}\left(\partial^{\rho} H^{\mu v}\right) u^{\alpha}\left(\partial^{\sigma} \tilde{u}_{v}\right)+c_{7}^{(11)} \epsilon_{\mu \rho \alpha \beta}\left(\partial^{\rho} H^{\mu v}\right) u^{\alpha}\left(\partial_{\nu} \tilde{u}^{\beta}\right) \\
& +c_{8}^{(11)} \epsilon_{\mu \sigma \alpha \beta}\left(\partial_{\nu} H^{\mu \nu}\right) u^{\alpha}\left(\partial^{\sigma} \tilde{u}^{\beta}\right) \\
& T^{(12)}=c_{1}^{(12)}\left(\partial^{\rho} \partial_{\nu} H^{\mu \nu}\right) u_{\mu} \tilde{u}_{\rho}+c_{2}^{(12)}\left(\partial^{\rho} \partial_{\nu} H^{\mu \nu}\right) u_{\rho} \tilde{u}_{\mu}+c_{3}^{(12)}\left(\partial_{\mu} \partial_{\nu} H^{\mu \nu}\right) u^{\rho} \tilde{u}_{\rho} \\
& +c_{4}^{(12)} \epsilon_{\mu \rho \alpha \beta}\left(\partial^{\rho} \partial_{v} H^{\mu \nu}\right) u^{\alpha} \tilde{u}^{\beta} \\
& T^{(13)}=c_{1}^{(13)} H^{\mu \nu} u_{\mu}\left(\partial_{\nu} \partial_{\rho} \tilde{u}_{\rho}\right)+c_{2}^{(13)} H^{\mu \nu} u_{\rho}\left(\partial^{\rho} \partial_{\nu} \tilde{u}_{\mu}\right)+c_{3}^{(13)} H^{\mu \nu} u^{\rho}\left(\partial_{\mu} \partial_{\nu} \tilde{u}_{\rho}\right) \\
& +c_{4}^{(13)} \epsilon_{\mu \rho \alpha \beta} H^{\mu \nu} u^{\alpha}\left(\partial^{\rho} \partial_{\nu} \tilde{u}_{\beta}\right) \\
& T^{(14)}=c_{1}^{(14)}\left(\partial^{\mu} \Phi\right) u_{\mu}\left(\partial_{\rho} \tilde{u}^{\rho}\right)+c_{2}^{(14)}\left(\partial^{\mu} \Phi\right) u^{\rho}\left(\partial_{\rho} \tilde{u}_{\mu}\right) \\
& +c_{3}^{(14)} \epsilon_{\mu \nu \alpha \beta}\left(\partial^{\mu} \Phi\right) u^{\alpha}\left(\partial^{v} \tilde{u}^{\beta}\right) \\
& T^{(15)}=c^{(15)} \Phi u_{\mu}\left(\partial^{\mu} \partial^{v} \tilde{u}_{\nu}\right) \\
& T^{(16)}=c^{(15)}\left(\partial^{\mu} \partial^{\nu} \Phi\right) u_{\mu} \tilde{u}_{\nu} ;
\end{aligned}
$$

then we have the terms containing at least one factor $v_{\mu}$ namely:

$$
\begin{aligned}
& U^{(1)}=d^{(1)} H^{\mu v} v_{\mu} v_{v} \\
& U^{(2)}=d^{(2)} \Phi v^{\mu} v_{\mu} \\
& U^{(3)}=d_{1}^{(3)} H^{\mu v}\left(\partial_{\alpha} H_{\mu \nu}\right) A^{\alpha}+d_{2}^{(3)} H^{\mu v}\left(\partial^{\alpha} H_{\mu \alpha}\right) v_{v} \\
& +d_{3}^{(3)} H^{\mu v}\left(\partial_{\nu} H_{\mu \alpha}\right) v^{\alpha}+d_{4}^{(3)} \epsilon_{\mu \rho \alpha \beta} H^{\mu v}\left(\partial^{\alpha} H^{\rho}{ }_{\nu}\right) v^{\beta} \\
& U^{(4)}=d^{(4)} H^{\mu v}\left(\partial_{\mu} \Phi\right) v_{v} \\
& U^{(5)}=d^{(5)} \Phi\left(\partial^{v} H_{\mu \nu}\right) v_{\mu} \\
& U^{(6)}=d^{(6)} \Phi\left(\partial^{\alpha} \Phi\right) v_{\alpha} \\
& U^{(7)}=d_{1}^{(7)}\left(\partial_{\alpha} v^{\alpha}\right) u_{\mu} \tilde{u}^{\mu}+d_{2}^{(7)}\left(\partial_{\mu} v_{\nu}\right) u^{\mu} \tilde{u}^{\nu}+d_{3}^{(7)}\left(\partial_{\nu} v_{\mu}\right) u^{\mu} \tilde{u}^{\nu} \\
& +d_{4}^{(7)} \epsilon_{\mu \nu \alpha \beta}\left(\partial^{\alpha} v^{\beta}\right) u^{\mu} \tilde{u}^{v} \\
& U^{(8)}=d_{1}^{(8)} v^{\alpha} u^{\mu}\left(\partial_{\alpha} \tilde{u}_{\mu}\right)+d_{2}^{(8)} v_{\mu} u^{\mu}\left(\partial_{\nu} \tilde{u}^{v}\right)+d_{3}^{(8)} v^{v} u^{\mu}\left(\partial_{\mu} \tilde{u}_{v}\right) \\
& +d_{4}^{(8)} \epsilon_{\mu \nu \alpha \beta} v^{\alpha} u^{\mu}\left(\partial^{\beta} \tilde{u}^{v}\right) \\
& U^{(9)}=d^{(9)} v_{\mu} v_{v}\left(\partial^{\mu} v^{v}\right) \\
& U^{(10)}=d_{1}^{(10)} H^{\mu \nu}\left(\partial_{\mu} v_{\alpha}\right)\left(\partial_{\nu} v^{\alpha}\right)+d_{2}^{(10)} H^{\mu \nu}\left(\partial_{\mu} v_{\nu}\right)\left(\partial_{\beta} v^{\beta}\right) \\
& +d_{3}^{(10)} H^{\mu \nu}\left(\partial_{\mu} v_{\rho}\right)\left(\partial^{\rho} v_{\nu}\right) \\
& +d_{4}^{(10)} \epsilon_{\mu \rho \alpha \beta} H^{\mu \nu}\left(\partial^{\rho} v_{\nu}\right)\left(\partial^{\alpha} v^{\beta}\right)+d_{5}^{(10)} \epsilon_{\mu \rho \alpha \beta} H^{\mu \nu}\left(\partial^{\rho} v^{\alpha}\right)\left(\partial_{\nu} v^{\beta}\right) \\
& U^{(11)}=d_{1}^{(11)} H^{\mu \nu} v^{\alpha}\left(\partial_{\mu} \partial_{\nu} v_{\alpha}\right)+d_{2}^{(11)} H^{\mu \nu} v_{\nu}\left(\partial_{\mu} \partial_{\beta} v^{\beta}\right)+d_{3}^{(11)} H^{\mu \nu} v^{\rho}\left(\partial_{\mu} \partial_{\rho} v_{\nu}\right) \\
& +d_{4}^{(11)} \epsilon_{\mu \alpha \rho \beta} H^{\mu v} v^{\alpha}\left(\partial^{\rho} \partial_{\nu} v^{\beta}\right)
\end{aligned}
$$




$$
\begin{aligned}
U^{(12)}= & d_{1}^{(12)} \Phi\left(\partial_{\alpha} v^{\alpha}\right)^{2}+d_{2}^{(12)} \Phi\left(\partial^{\mu} v^{\alpha}\right)\left(\partial_{\alpha} v_{\mu}\right) \\
& +d_{3}^{(12)} \epsilon_{\mu \nu \alpha \beta} \Phi\left(\partial^{\mu} v^{\alpha}\right)\left(\partial^{v} v^{\beta}\right) \\
U^{(13)}= & d^{(13)} \Phi v^{\alpha}\left(\partial_{\alpha} \partial_{\beta} v^{\beta}\right) \\
U^{(14)}= & d_{1}^{(14)} v^{\alpha}\left(\partial^{\beta} v_{\beta}\right)\left(\partial_{\alpha} \partial_{\nu} v^{\nu}\right)+d_{2}^{(14)} v^{\alpha}\left(\partial_{\alpha} v_{\beta}\right)\left(\partial^{\beta} \partial^{v} v_{v}\right) \\
& +d_{3}^{(14)} \epsilon_{\mu \nu \alpha \beta} v^{\alpha}\left(\partial^{\mu} v_{\beta}\right)\left(\partial^{v} \partial^{\rho} v_{\rho}\right)+d_{4}^{(14)} \epsilon_{\mu \nu \alpha \beta} v_{\rho}\left(\partial^{\mu} v^{\beta}\right)\left(\partial^{v} \partial^{\rho} v^{\alpha}\right) \\
& +d_{5}^{(14)} \epsilon_{\mu \nu \alpha \beta} v^{\alpha}\left(\partial^{\mu} v^{\rho}\right)\left(\partial^{v} \partial_{\rho} v^{\beta}\right) \\
U^{(15)}= & d^{(15)} v^{\alpha} v^{\beta}\left(\partial_{\alpha} \partial_{\beta} \partial_{\mu} v^{\mu}\right) .
\end{aligned}
$$

We have discarded a lot of terms because up to a total derivative they are of the type already considered. As a general strategy, we have eliminated all terms with derivatives on the ghost fields $u_{\mu}$. It is somewhat more complicated to prove that one can make $c_{3}^{(6)}=0$ if one subtracts a total divergence and redefines $c_{4}^{(6)}, c_{5}^{(6)}, c_{6}^{(6)}$, and $c_{9}^{(6)}=0$ if one subtract a total divergence and redefines $c_{7}^{(6)}, c_{8}^{(6)}$.

Also because

$$
\begin{aligned}
\left(\partial^{2}+m_{j}^{2}\right) f_{j}= & 0, \quad j=1,2,3 \rightarrow\left(\partial^{\mu} f_{1}\right)\left(\partial_{\mu} f_{2}\right) f_{3} \\
= & \frac{1}{2}\left(m_{1}^{2}+m_{2}^{2}-m_{3}^{2}\right) f_{1} f_{2} f_{3}+\frac{1}{2} \partial_{\mu}\left[\left(\partial^{\mu} f_{1}\right) f_{2} f_{3}\right. \\
& \left.+f_{1}\left(\partial^{\mu} f_{2}\right) f_{3}-f_{1} f_{2}\left(\partial^{\mu} f_{3}\right)\right]
\end{aligned}
$$

we can eliminate many terms by subtracting a total divergence and redefining other terms of lower canonical dimension.

Now we can put to zero some of the constants above if we subtract from $T$ a coboundary i.e. an expression of the form $d_{Q} B$ where we take $B$ to be a Wick polynomial with the following properties:

$$
\begin{aligned}
\mathcal{U}_{g} B & =B \mathcal{U}_{g}, \quad \forall g \in \mathcal{P} \\
g h(B) & =-1 \\
2 & \leq \operatorname{deg}(T) \leq 4 .
\end{aligned}
$$

As a result one take equal to zero the following coefficients:

$$
\begin{aligned}
& c_{2}^{(6)}, c_{4}^{(6)}, c_{5}^{(6)}, c_{7}^{(6)}, c_{1}^{(7)}, c_{2}^{(13)}, c^{(15)}, c_{1}^{(12)}, c_{2}^{(12)}, c_{3}^{(12)}, c_{4}^{(12)} d_{2}^{(3)}, \\
& \quad d^{(5)}, d_{1}^{(7)}, d_{2}^{(7)}, d_{3}^{(7)}, d_{4}^{(7)} .
\end{aligned}
$$

One can count that we are left with 37 coefficients of type $c^{(j)}$ and 23 coefficients of type $d^{(j)}$. So we have 60 free parameters which should be fixed by the condition of gauge invariance. 
Now one considers the coupling

$$
T=\sum_{j=1}^{16} T^{(j)}+\sum_{j=1}^{13} U^{(j)}
$$

and computes the expression $d_{Q} T$. It is convenient to follow the same strategy as above and eliminate, up to a divergence, the derivatives on the ghost fields $u_{\mu}$. One gets by a straightforward but tedious computation the following result:

$$
d_{Q} T=i \partial_{\mu} X^{\mu}+i u^{\mu} Y_{\mu}+i Z_{4}+i Z_{6}
$$

where the expressions $X^{\mu}, Y^{\mu}$ do not contain ghost fields and the expressions $Z_{j}$ are tri-linear in the ghost fields of canonical dimension $j=4,6$. We now impose the gauge invariance condition (2.46). It is sufficient to take $T^{\mu}$ a tri-linear expression in the fields $H_{\mu \nu}, \Phi, u_{\mu}, \tilde{u}_{\mu}, A_{\mu}$ verifying the following conditions:

$$
\begin{aligned}
\mathcal{U}_{g} T^{\mu} \mathcal{U}_{g}^{-1} & =\Lambda^{\mu \nu} T_{\nu}, \quad \forall g \in \mathcal{P} \\
g h\left(T^{\mu}\right) & =1 \\
3 & \leq \operatorname{deg}\left(T^{\mu}\right) \leq 5 .
\end{aligned}
$$

If we define

$$
\tilde{T}^{\mu} \equiv T^{\mu}-X^{\mu},
$$

then we get from (2.46) and (3.11):

$$
u^{\mu} Y_{\mu}+Z_{4}+Z_{6}=\partial_{\mu} \tilde{T}^{\mu} .
$$

The generic form for $\tilde{T}^{\mu}$ is

$$
\begin{aligned}
\tilde{T}^{\mu}= & u_{v} T^{\mu \nu}+\left(\partial_{\rho} u_{v}\right) T^{\mu \nu \rho}+\left(\partial_{\rho} \partial_{\sigma} u_{\nu}\right) T^{\mu \nu \rho \sigma} \\
& +d_{0} u^{\mu} u^{\nu} \tilde{u}_{v}+d_{1} \epsilon^{\mu \nu \rho \sigma} u_{\nu} u_{\rho} \tilde{u}_{\sigma}+S^{\mu}
\end{aligned}
$$

where the expressions $T^{\mu \nu}, T^{\mu \nu \rho}, T^{\mu \nu \rho \sigma}$ are bi-linear in the fields $H_{\mu \nu}, \Phi, A_{\mu}$, the expression $S^{\mu}$ is tri-linear in the ghost fields and of canonical dimension 5 $; d_{0}, d_{1}$ are some constants. One substitutes everything in equation (3.14) and obtains that $Y_{\mu}$ has the generic form

$$
Y_{\mu}=\frac{1}{2}\left(\partial^{2}+m^{2}\right) Z_{\mu}
$$

where $Z_{\mu}$ is a Wick polynomial bilinear in the fields $H_{\mu \nu}, \Phi, A_{\mu}$ and verifies the following conditions:

$$
\begin{aligned}
\mathcal{U}_{g} Z^{\mu} \mathcal{U}_{g}^{-1} & =\Lambda^{\mu \nu} Z_{\nu}, \quad \forall g \in \mathcal{P} \\
g h\left(Z^{\mu}\right) & =0 \\
2 & \leq \operatorname{deg}\left(Z^{\mu}\right) \leq 3 .
\end{aligned}
$$


If one considers the generic form of $Z_{\mu}$ then one finds out that the non-zero coefficients form the expression of $T$ are:

$$
\begin{aligned}
c^{(1)} & =\frac{2}{3} m^{2} a-\frac{1}{3} m^{2} b \quad c^{(2)}=\frac{1}{2} m^{2} a \quad c^{(3)}=-\frac{3}{4} m^{2} a \quad c^{(4)}=-m^{2} b \quad c^{(5)} \\
& =-m^{2} a \\
c_{1}^{(6)} & =-2 a \quad c_{6}^{(6)}=-4 a \quad c_{2}^{(7)}=2 a \quad c^{(8)}=4 a \quad c^{(9)}=a \\
c^{(10)} & =a \quad c_{2}^{(11)}=4 a \quad c_{3}^{(11)}=4 a \quad c_{3}^{(13)}=4 a \quad c_{2}^{(14)}=2 a \\
d^{(1)} & =\frac{3}{2} m^{2} b \quad d^{(2)}=m^{2} a \quad d_{1}^{(3)}=m b \quad d_{3}^{(3)}=-2 m b \quad d^{(4)}=m b \\
d_{1}^{(7)} & =m b \quad d_{2}^{(7)}=-4 m a-\frac{1}{2} m b \quad d_{3}^{(7)}=m b \quad d_{1}^{(8)}=m b \quad d_{2}^{(8)}=m b \quad d_{3}^{(8)} \\
& =-\frac{1}{2} m b \\
d^{(9)} & =2 m b \quad d_{1}^{(10)}=4 a-b \quad d_{2}^{(10)}=2 b \quad d_{3}^{(10)}=-2 b
\end{aligned}
$$

here $a, b \in \mathbb{R}$ are arbitrary parameters. Considering the terms tri-linear in the ghost fields from (3.14) we get

$$
d_{0}=-2 m^{2} a-\frac{3}{4} m^{2} b \quad d_{1}=0
$$

so we obtain the result from the statement.

The solution $T^{(a)}$ gives in the massless limit the usual gravity theory [29] plus the new term $4 H^{\mu \nu}\left(\partial_{\mu} v_{\rho}\right)\left(\partial_{\nu} v^{\rho}\right)$. The usual choice is $a=-\frac{1}{4}$.

We have considered in the previous theorem only the terms which are tri-linear in the fields. In principle, one should consider also the terms which are of degree 4 and 5 in the fields. It is plausible that, as in the case of Yang-Mills fields, one can obtain that these terms should be null as a consequence of the first order gauge invariance condition.

One can re-express $T^{(a)}$ using the variables from (2.13); we have

Proposition 3.2 In the variables

$$
h_{\mu \nu} \equiv H_{\mu \nu}+\frac{1}{2} \eta_{\mu \nu} \Phi \quad h \equiv h_{\mu}^{\mu}=2 \Phi
$$

the expression $T^{(a)}$ from the preceding theorem is equivalent to:

$$
\begin{aligned}
T= & h^{\mu \nu}\left(\partial_{\mu} h\right)\left(\partial_{\nu} h\right)-2 h^{\mu \nu}\left(\partial_{\mu} h_{\rho \sigma}\right)\left(\partial_{\nu} h^{\rho \sigma}\right)-4 h_{\mu \nu}\left(\partial_{\rho} h^{\mu \sigma}\right)\left(\partial_{\sigma} h^{\nu \rho}\right) \\
& -2 h^{\mu \nu}\left(\partial_{\rho} h_{\mu \nu}\right)\left(\partial^{\rho} h\right)+4 h^{\mu \nu}\left(\partial_{\sigma} h_{\mu \rho}\right)\left(\partial^{\sigma} h_{\nu}{ }^{\rho}\right) \\
& +4\left(\partial_{\mu} h^{\mu \nu}\right) u^{\rho}\left(\partial_{\rho} \tilde{u}_{\nu}\right)-4 h^{\mu \nu}\left(\partial_{\mu} u^{\rho}\right)\left(\partial_{\nu} \tilde{u}_{\rho}\right)+4 h^{\mu \nu}\left(\partial_{\mu} v_{\rho}\right)\left(\partial_{\nu} v^{\rho}\right) \\
& -4 m\left(\partial_{\mu} v_{\nu}\right) u^{\mu} \tilde{u}^{v}+m^{2}\left(-\frac{4}{3} h^{\mu \nu} h_{\mu \rho} h_{\nu}{ }^{\rho}+h^{\mu \nu} h_{\mu \nu} h-\frac{1}{6} h^{3}\right) .
\end{aligned}
$$


In these conditions one can take in (2.46)

$$
\begin{aligned}
T_{\mu}^{(a)}= & u^{\nu}\left[-\left(\partial_{\mu} h\right)\left(\partial_{\nu} h\right)+2\left(\partial_{\mu} h_{\rho \sigma}\right)\left(\partial_{\nu} h^{\rho \sigma}\right)-4\left(\partial_{\rho} h^{\rho \sigma}\right)\left(\partial_{\nu} h_{\mu \sigma}\right)\right. \\
& \left.+4\left(\partial_{\nu} h^{\rho \sigma}\right)\left(\partial_{\rho} h_{\mu \sigma}\right)-4\left(\partial_{\mu} v_{\rho}\right)\left(\partial_{\nu} v^{\rho}\right)\right] \\
& +u_{\mu}\left[\frac{1}{2}\left(\partial_{\nu} h\right)\left(\partial^{v} h\right)-\left(\partial_{\nu} h_{\rho \sigma}\right)\left(\partial^{\nu} h^{\rho \sigma}\right)-2\left(\partial_{\rho} h_{\nu \sigma}\right)\left(\partial^{\sigma} h^{\nu \rho}\right)\right. \\
& \left.+2\left(\partial_{\nu} v_{\rho}\right)\left(\partial^{v} v^{\rho}\right)\right] \\
& +\left(\partial^{\rho} u^{\nu}\right)\left[4 h_{\rho \sigma}\left(\partial^{\sigma} h_{\mu \nu}\right)+4 h_{\mu \sigma}\left(\partial_{\nu} h_{\rho}^{\sigma}\right)+2 h_{\nu \rho}\left(\partial_{\mu} h\right)-4 h_{\rho \sigma}\left(\partial_{\mu} h^{\sigma}{ }_{\nu}\right)\right] \\
& -4\left(\partial^{\rho} u_{\mu}\right) h^{\nu \sigma}\left(\partial_{\nu} h_{\rho \sigma}\right)+\left(\partial^{\rho} u_{\rho}\right)\left[-h\left(\partial_{\mu} h\right)+2 h^{\nu \sigma}\left(\partial_{\mu} h_{\nu \sigma}\right)\right] \\
& +2\left[u^{\nu}\left(\partial_{\mu} u^{\rho}\right)\left(\partial_{\nu} \tilde{u}_{\rho}\right)+u^{\nu}\left(\partial_{\nu} u^{\rho}\right)\left(\partial_{\mu} \tilde{u}_{\rho}\right)-u_{\mu}\left(\partial^{\nu} u^{\rho}\right)\left(\partial_{\nu} \tilde{u}_{\rho}\right)\right] \\
& +m^{2} u_{\mu}\left(h^{\nu \rho} h_{\nu \rho}-\frac{1}{2} h^{2}\right) .
\end{aligned}
$$

Proof It is convenient to start from the expression $T$ above and make the substitution $H_{\mu \nu} \equiv h_{\mu \nu}-\frac{1}{2} \eta_{\mu \nu} \Phi \quad h \equiv 2 \Phi$. Then one makes the transformations described at the beginning of the Proof of the preceding theorem, namely we get rid of derivatives appearing on $u_{\rho}$ subtraction total divergences. We also note that the fourth and the fifth terms from the expression of $T$ can be eliminated if we use the identity (3.8) but have been included such that the linear approximation of the Hilbert Lagrangian is reproduced [29]. We obtain the expression $T^{(a)}$ from the statement of the theorem. (2.13).

In the same way, one can also re-express $T^{(b)}$ using the variables from from

\section{Gauge invariance in the second order of perturbation theory}

In this section, we consider the second order gauge invariance. For this we must construct the chronological products $T(x, y)$ and $T_{\mu}(x, y)$ such that the identity (3.14) is verified. The construction procedure is well-known: one computes first the corresponding causal commutators $[T(x), T(y)]$ and $\left[T_{\mu}(x), T(y)\right]$ and makes the substitution $D_{m}(x-y) \mapsto D_{m}^{F}(x-y)$ i.e. one substitutes the causal PauliJordan distribution by the corresponding Feynman propagator and similar substitutions for the loop graphs; one obtains the expressions $T^{F}(x, y)$ and $T_{\mu}^{F}(x, y)$ which verify all Bogoliubov axioms but might spoil second order gauge invariance. To restore it we must annihilate some anomalies and make finite renormalizations. These finite renormalizations must also preserve the power counting theorem which in this case says [29] that the expressions $T(x, y)$ and $T^{\mu}(x, y)$ should be of the form

$$
T(x, y)=\sum_{j} t_{j}(x-y) W_{j}(x, y)
$$

where $W_{j}$ are Wick polynomials and $t_{j}$ are distributions such that one has

$$
\omega\left(t_{j}\right)+\operatorname{deg}\left(W_{j}\right) \leq 6 .
$$


The origin of the anomalies is explained in $[12,29]$. One starts from the identity

$$
d_{Q}[T(x), T(y)]=i \frac{\partial}{\partial x^{\mu}}\left[T^{\mu}(x), T(y)\right]+i \frac{\partial}{\partial y^{\mu}}\left[T(x), T^{\mu}(y)\right]
$$

which follows from first order gauge invariance. If one substitutes expressions of the type (4.1) for the causal commutators

$$
D(x, y)=\sum_{j} d_{j}(x-y) W_{j}(x, y)
$$

then the preceding identity reduces to some identities verified by the distributions $d_{j}$. When we make the causal splitting of the these distributions, preserving the degree of singularity, some of these identities are lost and we get anomalies. From tree Feynman graphs we get the identity

$$
\left(\partial^{2}+m^{2}\right) D_{m}=0
$$

which cannot be split causally preserving the degree of singularity; indeed it is well known that

$$
\left(\partial^{2}+m^{2}\right) D_{m}^{F}=\delta(x-y) .
$$

From loop graphs, we get the identities

$$
\begin{aligned}
& \partial_{\mu} D_{m_{1}, m_{2}}^{\mu}=-m_{2}^{2} D_{m_{1}, m_{2}}+\eta_{\mu \nu} D_{m_{1}, m_{2}}^{\mu \nu} \\
& \partial_{\mu} D_{m_{1}, m_{2}}^{\mu \nu}=-m_{1}^{2} D_{m_{2}, m_{1}}^{\nu}+\tilde{D}_{m_{1}, m_{2}}^{\mu} \\
& \partial_{\mu} \tilde{D}_{m_{1}, m_{2}}^{\mu \nu}=-m_{2}^{2} D_{m_{1}, m_{2}}^{\nu}+\tilde{D}_{m_{1}, m_{2}}^{\mu}
\end{aligned}
$$

where we have introduced the following distributions with causal support:

$$
\begin{aligned}
D_{m_{1}, m_{2}} & \equiv D_{m_{1}}^{(+)} D_{m_{2}}^{(+)}-D_{m_{1}}^{(-)} D_{m_{2}}^{(-)} \\
D_{m_{1}, m_{2}}^{\mu} & \equiv D_{m_{1}}^{(+)} \partial^{\mu} D_{m_{2}}^{(+)}-D_{m_{1}}^{(-)} \partial^{\mu} D_{m_{2}}^{(-)} \\
D_{m_{1}, m_{2}}^{\mu \nu} & \equiv \partial^{\mu} D_{m_{1}}^{(+)} \partial^{v} D_{m_{2}}^{(+)}-\partial^{\mu} D_{m_{1}}^{(-)} \partial^{v} D_{m_{2}}^{(-)} \\
\tilde{D}_{m_{1}, m_{2}}^{\mu \nu} & \equiv D_{m_{1}}^{(+)} \partial^{\mu} \partial^{v} D_{m_{2}}^{(+)}-D_{m_{1}}^{(-)} \partial^{\mu} \partial^{v} D_{m_{2}}^{(-)} . \\
\tilde{D}_{m_{1}, m_{2}}^{\mu} & \equiv \partial_{\nu} D_{m_{1}}^{(+)} \partial^{\mu} \partial^{v} D_{m_{2}}^{(+)}-\partial_{\nu} D_{m_{1}}^{(-)} \partial^{\mu} \partial^{v} D_{m_{2}}^{(-)} .
\end{aligned}
$$

It is easy to prove that one can use the arbitrariness of the causal splitting of these distributions such that one can eliminate all anomalies of the Ward identities (4.7)-(4.9). So it follows that only tree Feynman graphs can produce anomalies. tion.

We apply this strategy to the quantum gravity model from the preceding sec-

First we consider the theory given by the interaction Lagrangian $T=T^{(a)}$. 
Theorem 4.1 The Lagrangian $T=T^{(a)}$ gives a theory gauge invariant in the second order of the perturbation theory if we perform convenient finite renormalizations of the second-order chronological products

$$
\begin{aligned}
T(x, y)= & T^{F}(x, y)+i \delta(x-y) N(x) \quad T_{\mu}(x, y)=T_{\mu}^{F}(x, y) \\
& +i \delta(x-y) N_{\mu}(x)
\end{aligned}
$$

where $N$ and $N^{\mu}$ are some Wick polynomials. In particular:

$$
\begin{aligned}
& N=16 h^{\mu v} h^{\rho \sigma}\left(\partial_{\rho} h_{\mu \nu}\right)\left(\partial_{\sigma} h\right)-8 h^{\mu v} h^{\rho \sigma}\left(\partial_{\alpha} h_{\mu \nu}\right)\left(\partial^{\alpha} h_{\rho \sigma}\right) \\
& -32 h^{\mu v} h_{v \rho}\left(\partial^{\alpha} h^{\rho \beta}\right)\left(\partial_{\beta} h_{\mu \alpha}\right) \\
& -32 h^{\mu v} h^{\rho \sigma}\left(\partial_{\mu} h^{\rho \alpha}\right)\left(\partial_{\nu} h_{\sigma}{ }^{\alpha}\right)+32 h^{\mu v} h_{\nu \rho}\left(\partial^{\alpha} h_{\mu \beta}\right)\left(\partial_{\alpha} h^{\rho \beta}\right) \\
& +16 h^{\mu v} h^{\rho \sigma}\left(\partial_{\alpha} h_{\mu \rho}\right)\left(\partial^{\alpha} h_{v \sigma}\right) \\
& -16 h^{\mu v} h_{v \rho}\left(\partial^{\alpha} h_{\mu}{ }^{\rho}\right)\left(\partial_{\alpha} h\right)+16 u^{\rho}\left(\partial_{\rho} \tilde{u}^{v}\right) u^{\sigma}\left(\partial_{\sigma} \tilde{u}_{v}\right) \\
& +2 m^{2}\left(\frac{1}{12} h^{4}-h^{\mu v} h_{\mu \nu} h^{2}+\frac{8}{3} h^{\mu v} h_{\nu \rho} h^{\rho}{ }_{\mu} h+h^{\mu v} h_{\mu \nu} h^{\rho \sigma} h_{\rho \sigma}\right. \\
& \left.-4 h^{\mu v} h_{\nu \rho} h_{\mu \sigma} h^{\rho \sigma}\right) \text {. }
\end{aligned}
$$

Proof As it is known, the first step is to compute the causal commutator $\left[T^{\mu}(x), T(y)\right]$. The anomalies are produced by two types of terms in $T^{\mu}(x)$ : (a) with the index $\mu$ appearing in a derivative $\partial_{\mu}$; (b) with the index $\mu$ appearing in the combination $h_{\mu \rho}$. Inspecting the expression from Proposition 3.2 we have an expression of the following type

$$
\begin{aligned}
T_{\mu}=T_{1}\left(\partial_{\mu} h\right)+T_{2}^{\alpha \beta}\left(\partial_{\mu} h_{\alpha \beta}\right)+ & T_{3}^{\nu}\left(\partial_{\mu} \tilde{u}_{\nu}\right)+T_{4}^{v}\left(\partial_{\mu} u_{\nu}\right)+T_{5}^{v}\left(\partial_{\mu} v_{\nu}\right) \\
& +S^{\nu \rho}\left(\partial_{\nu} h_{\mu \rho}\right)+S^{\rho} h_{\mu \rho}+\cdots
\end{aligned}
$$

where by.. we mean terms which do not produce anomalies and the expression $T_{j}^{\cdots}, S^{\cdots}$ must be obtained explicitly. One has to compute the commutator of $\partial^{\mu} h, \partial^{\mu} h^{\alpha \beta}, \partial^{\mu} \tilde{u}^{\rho}, \partial^{\mu} u^{\rho}, \partial^{\mu} A^{\rho}$ with the 12 linear independent Wick monomials which appear in the expression of the total coupling $T$. Using the causal (anti)commutation relations from Sect. 4 we get in the end

$$
\begin{aligned}
{\left[T^{\mu}(x), T(y)\right]=} & i \partial_{\mu} D_{m}(x-y) A(x, y) \\
& +i \partial_{\mu} \partial_{\nu} D_{m}(x-y) A^{\nu}(x, y)+\cdots
\end{aligned}
$$

where the expressions $A(x, y)$ and $A^{v}(x, y)$ must be explicitly obtained. From the preceding formula we get the anomaly as explained in the beginning of this section:

$$
a(x, y)=\delta(x-y) A(x)+\left[\partial_{\nu} \delta(x-y)\right] A^{\nu}(x, y)
$$

By integration by parts we can rewrite this as follows:

$$
a(x, y)=\delta(x-y) \mathcal{A}(x)-\partial_{\nu}^{y}\left[\delta(x-y) \mathcal{A}^{v}(y)\right]
$$

with

$$
\begin{aligned}
\mathcal{A}(x) & \equiv A(x)+\left.\partial_{v}^{y} A^{v}(x, y)\right|_{y=x} \\
\mathcal{A}^{v}(x) & \equiv A^{v}(x, x)
\end{aligned}
$$


The total anomaly comes from the two commutators $\left[T^{\mu}(x), T(y)\right]+$ $\left[T^{\mu}(y), T(x)\right]$ and is

$$
\mathcal{A}(x, y)=a(x, y)+a(y, x) .
$$

Now, the second-order gauge invariance condition (2.45) for $n=2$ is fulfilled iff one can write this anomaly as follows:

$$
\mathcal{A}(x, y)=2 i d_{Q} N(x, y)+\partial_{\mu}^{x} N^{\mu}(x, y)+\partial_{\mu}^{y} N^{\mu}(y, x)
$$

where $N(x, y)$ and $N^{\mu}(x, y)$ are quasi-local Wick polynomials. One can show rather easily that this condition is equivalent to:

$$
\mathcal{A}=i d_{Q} N+\partial_{\mu} N^{\mu}
$$

for some Wick polynomials (in one variable) $N$ and $N^{\mu}$ of ghost number 0 and resp. 1 ; here $\mathcal{A}$ is given by the first formula (4.17). From power counting considerations (4.2) one also has the limitations

$$
\operatorname{deg}(N), \operatorname{deg}\left(N^{\mu}\right) \leq 6 .
$$

The condition (4.20) is the basic condition which must be investigated. It is fulfilled if we take $N$ to be exactly the expression from the statement of the theorem.

Considering the general case (i.e. $b \neq 0$ ) one can prove that the condition of second order gauge invariance gives in fact $b=0$.

\section{Conclusions}

Now comes a surprise ! In our derivation of massive quantum gravity we have not used any classical Lagrangian, whatsoever. Instead, we have started from the free massive quantized asymptotic spin-2 tensor field, defined a gauge structure for it and deduced the coupling from first and second order perturbative gauge invariance. Nevertheless, we may ask the question whether there exists a classical Lagrangian $L$ in terms of the metric tensor

$$
g^{\mu v}=\eta^{\mu \nu}+\kappa \hat{h}^{\mu v}
$$

which, after expansion in powers of the gravitational constant $\kappa^{2}=32 \pi G$, leads to the coupling we have found, similarly as in the massless case ([29], Sect. 5.5). Note that in (5.1) the background metric must be Minkowskian because that is what we have assumed throughout, but $\hat{h}^{\mu v}$ is the massive spin-2 field.

There are not many candidates for the Lagrangian $L$. The right one is Einstein's Lagrangian with a cosmological constant $\Lambda$

$$
L_{E}=-\frac{2}{\kappa^{2}} \sqrt{-g}(R-2 \Lambda), \quad \kappa^{2}=32 \pi G,
$$

where $g=\operatorname{det}\left(g_{\mu \nu}\right)$. For the expansion in powers of $\kappa$ it is convenient to use the so-called Goldberg variables

$$
\tilde{g}^{\mu \nu}=\sqrt{-g} g^{\mu \nu}, \quad \tilde{g}_{\mu \nu}=(-g)^{-1 / 2} g_{\mu \nu} .
$$


Now we write this metric tensor as

$$
\begin{aligned}
\tilde{g}^{\mu \nu} & =\eta^{\mu \nu}+\kappa h^{\mu \nu} \\
\tilde{g}_{\mu \nu} & =\eta_{\mu \nu}-\kappa h_{\mu \nu}+\kappa^{2} h_{\mu \alpha} h^{\alpha}{ }_{\nu}-\kappa^{3} h_{\mu \alpha} h^{\alpha \beta} h_{\beta \nu}+\cdots \\
& =\eta_{\mu \lambda}\left(\delta_{v}^{\lambda}-\kappa h^{\lambda}{ }_{\nu}+\kappa^{2} h_{\cdot \alpha}^{\lambda} h_{\cdot \nu}^{\alpha}-\kappa^{3} h^{\lambda}{ }_{\alpha} h^{\alpha \beta} h_{\beta \nu}+\cdots\right),
\end{aligned}
$$

then we can expand the determinant $g$ and the Ricci scalar $R$ in (5.2) in powers of $\kappa$; details can be found in [29], Sect. 5.5.

The first four orders in $\kappa$, i.e. $O\left(\kappa^{-2}\right), \ldots O\left(\kappa^{2}\right)$ come out to be:

$$
\begin{aligned}
L_{E}= & \frac{4 \Lambda}{\kappa^{2}}+\frac{2}{\kappa}\left(\partial^{2} h-\partial_{\alpha} \partial_{\beta} h^{\alpha \beta}+\Lambda h\right) \\
& \left.+\frac{1}{2}\left(\partial_{\gamma} h_{\alpha \beta}\right)\left(\partial^{\gamma} h_{\alpha \beta}\right)-\frac{1}{2} \partial_{\gamma} h\right)\left(\partial^{\gamma} h\right)+\left(\partial_{\alpha} h^{\alpha \beta}\right)\left(\partial_{\beta} h\right)-\left(\partial_{\gamma} h^{\alpha \beta}\right)\left(\partial_{\alpha} h_{\beta}^{\gamma}\right) \\
& +\Lambda\left(\frac{1}{2} h^{2}-h^{\alpha \beta} h_{\alpha \beta}\right) \\
& +\kappa\left[L^{(1)}+4 \Lambda\left(\frac{1}{6} h^{\alpha \beta} h_{\beta \gamma} h_{\alpha}^{\gamma}-\frac{1}{8} h^{\alpha \beta} h_{\alpha \beta} h+\frac{1}{48} h^{3}\right)\right] \\
& +\kappa^{2}\left\{L^{(2)}+4 \Lambda\left[\frac{1}{32}\left(h^{\alpha \beta} h_{\alpha \beta}\right)^{2}+\frac{1}{12} h h^{\alpha \beta} h_{\beta \gamma} h_{\alpha}^{\gamma}-\frac{1}{32} h^{2} h^{\alpha \beta} h_{\alpha \beta}\right.\right. \\
& \left.\left.+\frac{1}{4 !} \frac{h^{4}}{16}-\frac{1}{8} h^{\alpha \beta} h_{\beta \gamma} h^{\gamma \nu} h_{\nu \alpha}\right]\right\}
\end{aligned}
$$

Here, the terms $L^{(1)}$ and $L^{(2)}$ without $\Lambda$ are the same as in the ordinary massless gravity:

$$
\begin{aligned}
L^{(1)} \equiv & -\frac{1}{4} h^{\alpha \beta}\left(\partial_{\alpha} h\right)\left(\partial_{\beta} h\right)+\frac{1}{2} h^{\mu \nu}\left(\partial_{\mu} h^{\alpha \beta}\right)\left(\partial_{\nu} h^{\alpha \beta}\right)+h^{\alpha \beta}\left(\partial_{\nu} h^{\alpha \mu}\right)\left(\partial_{\mu} h^{\beta \nu}\right) \\
& +\frac{1}{2} h^{\mu \nu}\left(\partial_{\alpha} h^{\mu \nu}\right)\left(\partial_{\alpha} h\right)-h^{\mu \nu}\left(\partial_{\rho} h^{\alpha \mu}\right)\left(\partial^{\rho} h^{\alpha \nu}\right) \\
L^{(2)} \equiv & -h_{\alpha \rho} h^{\rho}{ }_{\beta}\left(\partial_{\nu} h^{\alpha \mu}\right)\left(\partial_{\mu} h^{\beta \nu}\right)-\frac{1}{2} h_{\rho \beta} h^{\beta}{ }_{\gamma}\left(\partial_{\alpha} h^{\rho \gamma}\right)\left(\partial^{\alpha} h\right) \\
& -\frac{1}{4} h_{\mu \nu}\left(\partial_{\alpha} h^{\mu \nu}\right) h_{\rho \gamma}\left(\partial^{\alpha} h^{\rho \gamma}\right)+\frac{1}{2} h_{\mu \nu}\left(\partial_{\alpha} h^{\mu \nu}\right) h^{\alpha \beta}\left(\partial_{\beta} h\right) \\
& -h_{\alpha \rho}\left(\partial_{\mu} h^{\rho}{ }_{\gamma}\right)\left(\partial_{\nu} h^{\alpha \gamma}\right) h^{\mu \nu} \\
& +h_{\rho \beta} h^{\beta}{ }_{\gamma}\left(\partial_{\mu} h^{\alpha \rho}\right)\left(\partial_{\alpha} h_{\alpha}^{\gamma}\right)+\frac{1}{2} h_{\alpha \rho} h_{\beta \gamma}\left(\partial_{\mu} h^{\alpha \gamma}\right)\left(\partial^{\mu} h^{\beta \rho}\right) .
\end{aligned}
$$

The terms $O\left(\kappa^{-2}\right)$ and $O\left(\kappa^{-1}\right)$ in the Lagrangian (5.6) as they stand are meaningless: the constant $4 \Lambda / \kappa^{2}$ cannot be integrated over $d^{4} x$ and the term $O\left(\kappa^{-1}\right)$ linear in $h$ alone gives no consistent equation of motion. But the constant is trivially a divergence and can be dropped. The same is true for the term linear in $h$ if we take the equation of motion into account which follows from the term $O\left(\kappa^{0}\right)$ 
and quadratic in $h$

$$
\begin{aligned}
L^{(0)} \equiv & \frac{1}{2}\left(\partial^{\mu} h^{\alpha \beta}\right)\left(\partial_{\mu} h_{\alpha \beta}\right)-\left(\partial_{\mu} h^{\alpha \beta}\right)\left(\partial_{\beta} h_{\alpha}^{\mu}\right)-\frac{1}{4}\left(\partial_{\alpha} h\right)\left(\partial^{\alpha} h\right) \\
& +\Lambda\left(\frac{h^{2}}{2}-h^{\alpha \beta} h_{\alpha \beta}\right)
\end{aligned}
$$

As usual, this quadratic Lagrangian defines the free asymptotic theory. It gives the following Euler-Lagrange equation

$$
\partial^{2} h^{\alpha \beta}-\partial_{\mu}\left(\partial^{\beta} h^{\alpha \mu}+\partial^{\alpha} h^{\beta \mu}\right)-\frac{1}{2} \eta^{\alpha \beta} \partial^{2} h-\Lambda\left(\eta^{\alpha \beta} h-2 h^{\alpha \beta}\right)=0 .
$$

Taking the trace we find

$$
\partial_{\mu} \partial_{\alpha} h^{\alpha \mu}=-\frac{1}{2} \partial^{2} h-\Lambda h
$$

Differentiating (5.10) by $\partial_{\alpha}$ and substituting (5.11) we derive the Hilbert gauge condition

$$
\partial_{\alpha} h^{\alpha \beta}=0 .
$$

Then (5.11) reduces to the Klein-Gordon equation

$$
\partial^{2} h+2 \Lambda h=0
$$

and from (5.10) we obtain the Klein-Gordon equation for the tensor field

$$
\partial^{2} h^{\alpha \beta}+2 \Lambda h^{\alpha \beta}=0 .
$$

This means the graviton becomes massive with mass

$$
m^{2}=2 \Lambda \text {. }
$$

It follows from (5.12-13) that the term $O\left(\kappa^{-1}\right)$ linear in $h$ can be written as $\partial^{2} h / \kappa$. Since this is a divergence we have omitted this term, too. Taking the current value of $\Lambda$ one finds a tiny mass $\left(\approx 10^{-32} \mathrm{eV}\right)$ for the graviton, so that our massive theory passes all direct tests of general relativity. However, gravitational radiation requires a thorough investigation.

Now comes the main point. The cubic part $O\left(\kappa^{1}\right)$ in (5.6) should give the coupling. Using (5.15) this agrees exactly with the pure graviton coupling terms in (3.21), if we multiply with an overall factor -4 . Similarly, the pure graviton couplings in the quartic part $O\left(\kappa^{2}\right)$ agree with (4.12) without the ghost terms, if we multiply by 16 . This shows that our massive gravity is the quantum gauge theory corresponding to classical gravity with a cosmological term. But there seems to be an inconsistency in this classical expansion because the Minkowski background is not a solution of Einstein's equation with cosmological term. This solution is the de-Sitter or anti-de-Sitter metric. But locally all those metrics are Minkowskian. The quantum field theory we have constructed describes the quantum fluctuations around this local Minkowskian background. The classical theory is relevant for the large distances. Indeed, the cosmological constant and, hence, the mass of the graviton has only be observed on the largest astronomical scale (supernova 
data). One could also construct a quantum theory valid on all scales by quantizing the gravitational field on de-Sitter background, say, but this is another story. The resulting theory would not be universal because it depends on the chosen background. But locally it should agree with the theory constructed here.

In spin 1 gauge, theories with massive gauge fields a Higgs field is necessary to "generate" the masses. In the framework of quantum gauge theory it is simply needed to restore gauge invariance to second order [29]; first order gauge invariance holds without the Higgs couplings. It was a surprise for us when we found that massive gravity is gauge invariant to first and second order without a Higgs field. In a way, this is even disappointing because a gravitational Higgs field would be a nice candidate for the non-baryonic dark matter. It is still possible that a Higgs field is necessary in third order. Work in that direction is in progress.

A last remark is concerned with the mass zero limit of our theory. As noticed above, in the limit $m \rightarrow 0$ the bosonic ghost $v^{\mu}$ does not completely decouple from the graviton. In fact, the term $4 h^{\mu \nu}\left(\partial_{\mu} v_{\rho}\right)\left(\partial_{\nu} v^{\rho}\right)$ survives in (3.21). That means the resulting massless theory is not identical with usual quantum gravity as discussed in [29], for example. This leads to the conclusion that there exists at least two different quantum gauge theories which correspond to classical (massless) general relativity, one with an additional Bose field $v^{\mu}$ and one without.

\section{References}

1. Ashtekar, A.: Lectures on Non Perturbative Canonical Gravity. World Scientific (1991)

2. Arkani-Hamad, N.M., Georgi, H., Schwartz, M.D.: Effective field theory for massive gavitons and gravity in theory space. [hep-th/0210184]

3. Capper, D.M., Leibbrandt, G., Medrano, M.R.: Calculation of the graviton self-energy using dimensional regularization. Phys. Rev. D 8, 4320-4331 (1973)

4. Donoghue, J.F.: General relativity as an effective field theory. The leading quantum corrections. Phys. Rev. D 6, 3874-3888 (1994)

5. van Dam, H., Veltman, H.M.: Massive and massless yang-mills and gravitational fields. Nucl. Phys. B 22, 397-411 (1970)

6. Damour, T., Kogan, I.I., Papazoglou, A.: Spherically symmetric spacetimes in massive gravity. Phys. Rev. D 67 (2003) [064009:1-25]

7. Epstein, H., Glaser, V.: The rôle of locality in perturbation theory. Ann. Inst. H. Poincaré 19A, 211-295 (1973)

8. Feynman, R.P.: Quantum theory of gravitation. Acta Phys. Polonica XXIV, 679-722 (1963)

9. Glaser, V.: Electrodynamique Quantique, L'enseignement du 3e cycle de la physique en Suisse Romande (CICP). Semestre d'hiver 1972/73

10. Goldberg, J.N.: Conservation laws in general relativity. Phys. Rev. 111, 315-320 (1958)

11. Grigore, D.R.: On the uniqueness of the non-abelian gauge theories in the epstein-glaser approach to renormalization theory. Romanian Journ. Phys. 44, 853-913 (1999) [hepth/9806244]

12. Grigore, D.R.: The standard model and its generalisations in epstein-glaser approach to renormalisation theory. J. Phys. A 33, 8443-8476 (2000) [hep-th/9810078]

13. Grigore, D.R.: On the quantization of the linearized gravitational field. Class. Quant. Grav. 17, 319-344 (2000) [hep-th/9905190]

14. Grillo, N.: Some aspects of quantum gravity in the causal approach. the 4th workshop on Quantum field theory under the influence of external conditions. Leipzig, Germany, Sept. (1998) [hep-th/9903011]

15. Grillo, N.: Finite one-loop calculations in quantumm gravity: graviton self-energy, perturbative gauge invariance and Slavnov-Ward Identities. [hep-th/9912097]

16. Grillo, N.: Finite one-loop corrections and perturbative gauge invariance in quantumm gravity coupled to photon fields. [hep-th/9912114] 
17. Grillo, N.: Scalar matter coupled to quantumm gravity in the causal approach: finite oneloop calculations and pertutbative gauge invariance. [hep-th/9912128]

18. Gupta, S.N.: Einstein's other theories of gravitation. Rev. Mod. Phys. 29, 334-336 (1957)

19. Gupta, S.N.: Supplementary conditions in the quantized gravitational theory. Phys. Rev. 172, 1303-1307 (1968)

20. Goroff, M.H., Sagnotti, A.: The ultraviolet behaviour of einstein gravity. Nucl. Phys. B 266, 709-736 (1986)

21. Gabadadze, G., Shifman, M.: Softly massive gravity. [hep-th/0312289]

22. 't Hooft, G., Veltman, M.: One-loop divergences in the theory of gravitation. Ann. Inst. H. Poincaré XX, 69-94 (1974)

23. Kugo, T., Ojima, I.: Subsidiary conditions and physical $s$-matrix unitarity in indefinitemetric quantum gravitation theory. Nucl. Phys. B 144, 234-252 (1978)

24. Kugo, T., Ojima, I.: Local covariant operator formalism of non-abelian gauge theories and quark confinement problem. Suppl. of Progr. of Theor. Phys. 66, 1-130 (1979)

25. Luzy, M.A., Porrati, M., Rattazzi, R.: Strong interactions and stability in the DGP model. [hep-th/0303116]

26. Ogievetsky, V.I., Polubarinov, I.V.: Interacting field of spin 2 and the einstein equations. Ann. Phys. 35, 167-208 (1965)

27. Rattazzi, R., Scrucca, G.A., Struma, A.: Brane to brane gravity mediation and supersymmetry breaking. [hep-th/0305184]

28. Scharf, G.: Finite Quantum Electrodynamics: The Causal Approach, second edition. Springer (1995)

29. Scharf, G.: Quantum Gauge Theories: A True Ghost Story. J. Wiley (2001)

30. Schorn, I.: Gauge invariance of quantum gravity in the causal approach. Clas. Quantum Grav. 14, 653-669 (1997)

31. Scharf, G., Wellmann, M.: Quantum gravity from perturbative gauge invariance. Gen. Rel. Gravit. 33, 553 (2001). [hep-th/9903055]

32. Thiemann, T.: Lectures on loop quantum gravity. [gr-qc/0210094]

33. Thirring, W.: An alternative approach to the theory of gravitation. Ann. Phys. 16 (1961)

34. Vainshtein, A.I.: To the problem of nonvanishing gravitation mass. Phys. Lett. 39B, 393394 (1972)

35. van de Ven, A.E.M.: Two-loop quantum gravity. Nucl. Phys. B 378, 309-366 (1992)

36. Zaidi, S.A.A.: Self-energy of the graviton in second order. J. Phys. A: Math. Gen 24, 43254334 (1991)

37. Weinberg, S.: The Quantum Theory of Fields, 1 and 2. Cambridge Univ. Press (1995) 